\title{
A RESTRUCTURING OF HYPOTHALAMIC SYNAPSES IS ASSOCIATED WITH MOTHERHOOD ${ }^{1}$
}

\author{
JAMES D. HATTON ${ }^{2}$ AND MARK H. ELLISMAN \\ Department of Neurosciences, University of California at San Diego, La Jolla, California 92093 \\ Received August 18, 1981; Revised December 23, 1981; Accepted January 15, 1982
}

\begin{abstract}
Freeze-fracture analysis of the rat hypothalamic paraventricular nucleus reveals a variety of postsynaptic specializations distinguished both by particle size and by aggregate shape. One particular type of specialization is found only in replicas from females having born offspring. This suggests that, in association with motherhood, either existing synaptic specializations are modified or a new type of connection is formed.
\end{abstract}

Thin section analysis of the postsynaptic density in the cerebral cortex of the rat (Peters and KaisermanAbramof, 1969) and dog (Blomberg et al., 1978; Cohen et al., 1977; Cohen and Siekevitz, 1978) has shown this membrane specialization to be disk shaped, occasionally containing one or more holes or "perforations." Application of freeze-fracture techniques to the study of the postsynaptic specialization (PSS) reveals a cluster of particles on the protoplasmic fracture face of dendrites (Sandri et al., 1972). Where perforations in the postsynaptic density are seen in thin sections, particle-free zones in the particle aggregate are seen in freeze-fracture (Landis and Reese, 1974; Landis et al., 1974). Quantitative analysis of the size and densities of particles, as well as the presence or absence of particle-free zones, defines morphologically distinct classes of PSSs. It has been suggested that the different shapes of the PSSs represent physiologically distinct synapses or perhaps different structural forms of physiologically similar synapses (Landis and Reese, 1974). In fact, these differences may reflect the age and experience of an animal (Greenough et al., 1978).

\footnotetext{
' We thank U. V. Bouchard and D. D. Taitano for typing the manuscript, V. A. Bradshaw for inking the histograms, T. J. Deerinck for photographic assistance, and J. M. LeBeau for performing the statistical analyses. We also thank Dr. P. M. Groves for a thorough critique of an earlier draft of this paper. This work was supported in part by grants to M. H. E. from the National Institute of Neurological and Communicative Disorders and Stroke (Grant NS14718), the Muscular Dystrophy Association of America, and the National Multiple Sclerosis Society.

? To whom correspondence should be addressed at Department of Neurosciences, M-008, School of Medicine, University of California at San Diego, La Jolla, CA 92037.
}

In order to test the variable aspects of PSS morphology, we examined the synaptic connections of a system lending itself to several types of physiological manipulation. We report here that the synapses in the magnocellular paraventricular nucleus of the hypothalamus display PSSs with centrally located particle-free zones (annular PSSs) only in animals having been mothers at some time. If paraventricular nuclei from males or virgin females contain annular PSSs, their numbers are extremely low. Preliminary results of this study have been reported elsewhere (Hatton and Ellisman, 1980).

\section{Materials and Methods}

Sprague-Dawley rats in the following categories were prepared as described previously (Hatton and Ellisman, 1981): males, 80 to 100 days old, either normal or deprived of water for $24 \mathrm{hr}$; lactating mothers, 80 to 100 days old; retired female breeders, 300 to 350 days old; and virgin females, 30 to 40,80 to 100 (either normal or deprived of water for $24 \mathrm{hr}$ ), and 150 to 200 days old. In brief, each animal was anesthetized with pentobarbital and then perfused with a Ringer's solution followed by a paraformaldehyde/glutaraldehyde fixative. The magnocellular portions of the paraventricular nuclei were cored under a dissecting microscope from coronal slices approximately $750 \mu \mathrm{m}$ thick. All tissues were glycerinated, frozen, fractured, replicated, and examined under identical conditions.

Examinations and PSS classification were made without knowledge of group identification. Particle size measurements were made on prints at $\times 100,000$ with a $\times 10$ magnifying lens (total magnification, $\times 1,000,000$ ) containing a reticule. PSS area was determined using a 
compensating polar planimeter (Keuffel \& Esser model $62005)$. Statistical analyses were made using a Burroughs 7800 computer.

\section{Results and Discussion}

Particle aggregates representing PSSs were found in all replicas examined (see Table I). The majority of the aggregates, across hormonal groups, were of the irregular cluster type (that is, containing no particle-free zones). This type of aggregate was observed in all groups; however, an analysis of variance showed a significant difference in cluster number across groups $(F(5,60)=5.43 ; p$ $<0.05$ ). Comparison of means using the Scheffe test, $p$ $<0.05$, indicated that this difference is between male versus female groups. Irregular "perforated" PSSs also were noted in all cases, although they were always much fewer in number than the cluster type. In addition, the number of perforated PSSs, in contrast to the number of cluster PSSs, did not differ significantly across hormonal groups. Finally, in contrast to both perforated and cluster type PSSs, annular PSSs (distinguished from the irregular perforated PSSs by their circular aggregate shape and the central placement of the particle-free zones) were not observed in all hormonal groups; only lactating mothers and retired breeders showed the presence of this type of PSS. This difference was significant as indicated by an analysis of variance across groups $(F(5,60)=26.24 ; p$ $<0.01$ ) and the Scheffe test, $p<0.05$, comparing mothers versus non-mothers. These annuli were noted on the external fracture faces of both neuronal processes and apparent cell somas.

Particle size analysis showed that, although the most frequent particle size was the same across the three PSS categories, the annular aggregates contained larger numbers of large diameter particles (see Fig. 1). This suggests a different protein makeup for the annular PSS than for PSSs of other shapes.

Finally, although the largest cluster type PSSs were as large in area as the smaller perforated or annular PSSs, an analysis of variance showed a difference across PSS types with respect to diameter $(F(2,69)=6.81 ; p<0.01)$. Post hoc comparison of means using the Newman-Keuls test, $p<0.05$, showed that this difference was due solely to the significantly smaller area of the cluster type PSS: the diameter of cluster type PSSs differed significantly from both that of the perforated and that of the annular PSSs. Perforated and annular PSSs did not differ significantly from each other, suggesting that the increased area of the annular or perforated PSSs may be due to the area contributed by the particle-free perforations.

Freeze-fracture studies of the olfactory bulb (Landis et al., 1974) and cerebellar cortex (Landis and Reese, 1974) suggest that synapses which display PSSs as particle aggregates are excitatory synapses, while inhibitory synapses display no particle aggregates in the postsynaptic membrane. If postsynaptic aggregates do represent excitatory synapses, then the annular PSSs within the female paraventricular nucleus may identify a subset of excitatory synapses on those neurons. In fact, Carlin et al. (1980) recently showed that postsynaptic densities isolated from the cerebral cortex and midbrain of the dog, morphologically identified as Gray type I (putative excitatory) synapses, are generally annular in arrangement. As mentioned above, particle size analysis suggests that the annular PSSs have a different macromolecular composition than do other PSSs within this nucleus and, thus, may be associated with physiologically distinct synapses. The presence of annular PSSs in the paraventricular nuclei of retired female breeders also indicates that, once these specializations are formed, the state of having them persists past the immediate postpartum period.

Different populations of magnocellular neurons in the paraventricular nucleus synthesize the hormones vasopressin (antidiuretic hormone) and oxytocin, the latter of which plays an important role in lactation and parturition. To elucidate the role of the annular PSS in the paraventricular nucleus further, replicas from osmotically stressed virgin females of breeding age were examined. No annular PSSs were noted in these replicas, suggesting that diuretic stress is not related to the occurrence of these structures. Although circumstantial, this negative finding stimulates us to speculate that, among other possibilities, the annular PSSs are perhaps associated with oxytocin neurons. Determination of the molecular function and cellular participants in this particular synapse should provide new insight into the nature of synaptic plasticity and sexual dimorphism.

T'ABLE' I

Number of PSSs per replica of paraventricular nucleus by hormonal group and by PSS type

Shown here are the means of the number of PSSs per replica of the paraventricular nucleus by hormonal group (sex, age, and parturitic condition) and by PSS type. Each hormonal group is listed by condition and age in days; PSS types are listed with their respective mean square area over all hormonal groups, and $n$ represents the number of replicas examined for each hormonal group.

\begin{tabular}{|c|c|c|c|c|c|c|c|}
\hline \multirow[b]{2}{*}{ PSS Type } & \multirow[b]{2}{*}{ Mean Area } & \multicolumn{6}{|c|}{ Group } \\
\hline & & $\begin{array}{c}\text { G1 } \\
\text { Males } \\
80-100 \mathrm{~d}\end{array}$ & $\begin{array}{c}\text { G2 } \\
\text { Virgins } \\
30-40 d\end{array}$ & $\begin{array}{c}\text { G3 } \\
\text { Virgins } \\
80-100 \mathrm{~d}\end{array}$ & $\begin{array}{c}\mathrm{G} 4 \\
\text { Virgins } \\
150-200 \mathrm{~d}\end{array}$ & $\begin{array}{c}\text { G5 } \\
\text { Lactating Mothers } \\
80-100 \mathrm{~d}\end{array}$ & $\begin{array}{c}\mathrm{G} 6 \\
\text { Retired Breeders } \\
300-350 \mathrm{~d}\end{array}$ \\
\hline & $\mu m^{2}$ & & & & & & \\
\hline Cluster & 1.71 & 11.46 & 7.92 & 7.77 & 8.89 & 11.00 & 10.78 \\
\hline Perforated & 2.98 & 1.45 & 1.75 & 2.00 & 2.11 & 2.33 & 3.22 \\
\hline Annulus & 3.17 & 0.00 & 0.00 & 0.00 & 0.00 & 1.17 & 1.11 \\
\hline$n$ & & 11 & 12 & 13 & 9 & 12 & 9 \\
\hline
\end{tabular}



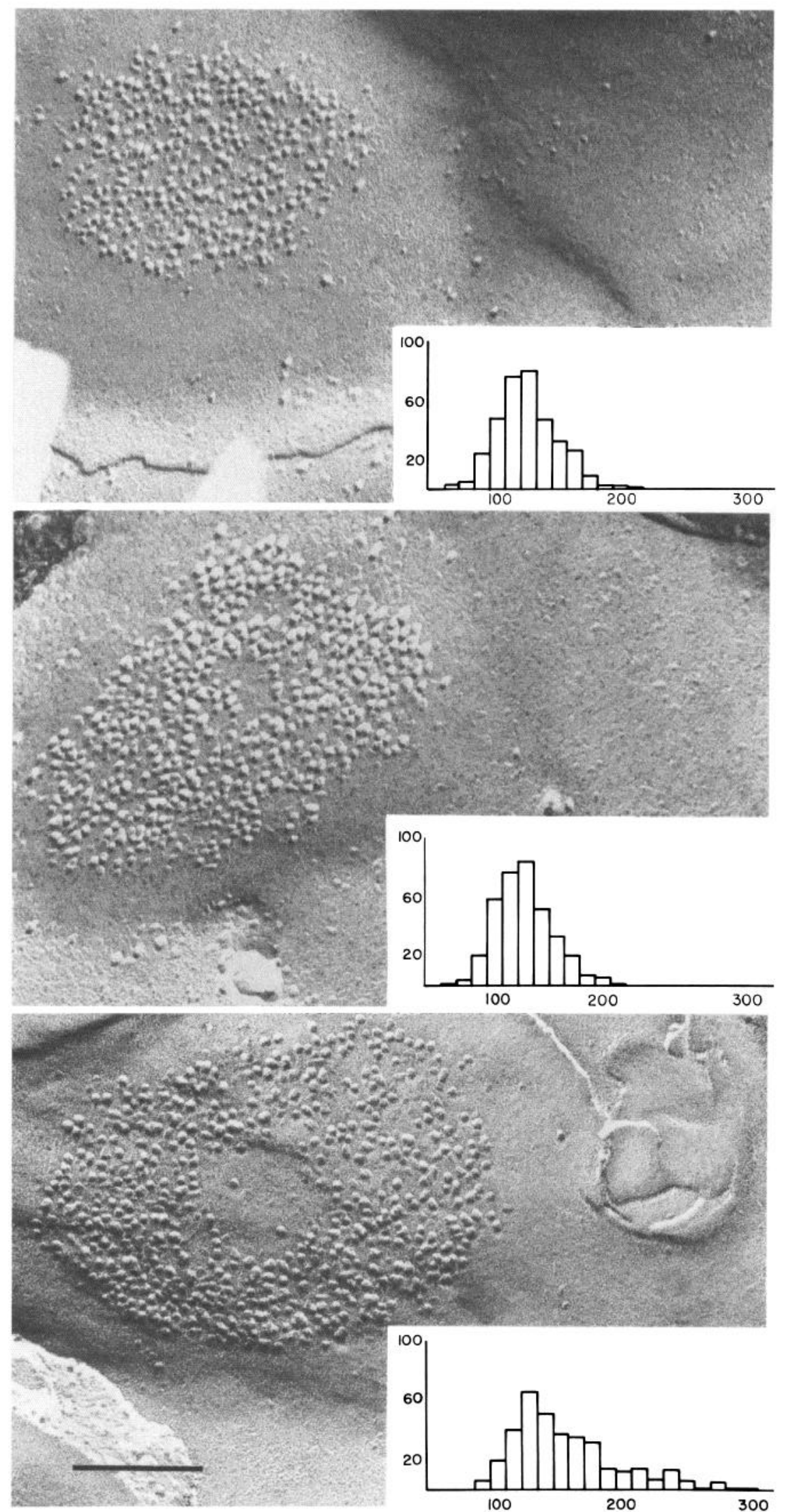

Figure 1. Synaptic types and particle sizes. Shown here are representative examples of the different classes of postsynaptic specializations (PSSs) found in the freeze-fractured paraventricular nucleus of the female rat. Top, Cluster type PSS; middle, irregular "perforated" PSS; bottom, annular PSS. The scale bar represents $0.2 \mu \mathrm{m}$ for all micrographs. Each inset represents a histogram of the sizes of the particles from that type of PSS, showing the number of particles counted versus particle size in Angstrom units. Each histogram is based on data from 360 particles randomly sampled from 18 PSSs in that particular class. Particle diameters were taken to be equivalent to the width of the shadow immediately behind the particle as measured perpendicular to the direction of platinum evaporation at a total magnification of $\times 1,000,000$. Although the most frequently occurring particle size is the same for all three classes, the annular PSSs contain larger numbers of large diameter particles, suggesting a different molecular makeup for this type of PSS. 


\section{References}

Blomberg, F., R. S. Cohen, and P. Siekevitz (1978) The structure of postsynaptic densities isolated from dog cerebral cortex. II. Characterization and arrangement of some of the major proteins within the structure. J. Cell Biol. 74: 204-225.

Carlin, R. K., D. J. Grab, R. S. Cohen, and P. Siekevitz (1980) Isolation and characterization of postsynaptic densities from various brain regions: Enrichment of different types of postsynaptic densities. J. Cell Biol. 86: 831-843.

Cohen, R. S., and P. Siekevitz (1978) Form of the postsynaptic density. A serial section study. J. Cell Biol. 78: 36-46.

Cohen, R. S., F. Blomberg, K. Berzins, and P. Siekevitz (1977) The structure of postsynaptic densities isolated from dog cerebral cortex. I. Overall morphology and protein composition. J. Cell Biol. 74: 181-203.

Greenough, W. T., R. W. West, and T. J. deVoogd (1978) Subsynaptic plate perforations: Changes with age and experience in the rat. Science 202: 1096-1098.

Hatton, J. D., and M. H. Ellisman (1980) A distinctly female postsynaptic specialization revealed by freeze-fracture analysis of the rat paraventricular nucleus. J. Cell Biol. 87: 82a.

Hatton, J. D., and M. H. Ellisman (1981) The distribution of orthogonal arrays and their relationship to intercellular junctions in neuroglia of the freeze-fractured hypothalamo-neurohypophysial system. Cell Tissue Res. 215: 309-323.

Landis, D. M. D., and T. S. Reese (1974) Differences in membrane structure between excitatory and inhibitory synapses in the cerebellar cortex. J. Comp. Neurol 155: 93-126.

Landis, D. M. D., T. S. Reese, and E. Raviola (1974) Differences in membrane structure between excitatory and inhibitory components of the reciprocal synapse in the olfactory bulb. J. Comp. Neurol. 155: 67-92.

Peters, A., and I. A. Kaiserman-Abramof (1969) The small pyramidal neuron of the rat cerebral cortex. The synapses upon dendritic spines. Z. Zellforsch. Mikrosk. Anat. 100: 487506.

Sandri, C., K. Akert, R. B. Livingston, and H. Moor (1972) Particle aggregations at specialized sites in freeze-etched postsynaptic membranes. Brain Res. 41: 1-16. 